\title{
EVOLUÇÃO DAS LINGUAGENS DE MARCAÇÃO: UM BREVE HISTÓRICO À LUZ DA ÁREA DE CIÊNCIA DA INFORMAÇÃO
}

\author{
EVOLUTION OF MARKUP LANGUAGES: A BRIEF \\ HISTORY IN LIGHT OF INFORMATION SCIENCE
}

\author{
Rogério Aparecido Sá Ramalho ${ }^{1}$ \\ Paulo George Miranda Martins ${ }^{2}$ \\ Janailton Lopes Sousa ${ }^{3}$
}

\section{RESUMO}

Introdução: As denominadas Linguagens de Marcação sempre tiveram relação direta com as práticas profissionais identificadas no campo da Ciência da informação, a partir do desenvolvimento do ambiente Web tais tecnologias ganharam maior destaque, impulsionado a criação de uma grande variedade de aplicações com diferentes características e propósitos. Objetivo: Apresentar um compêndio da evolução das linguagens de marcação, analisando tais tecnologias sob o enfoque da área de Ciência da Informação e descrever os conceitos e características das principais linguagens utilizadas na área. Metodologia: Revisão de literatura sobre evolução das linguagens de marcação, sob a perspectiva da Ciência da informação, abordagem qualitativa de natureza aplicada. Resultados: $O$ artigo apresenta uma breve revisão sobre a evolução das linguagens de marcação, contribuindo para uma maior discussão desta temática na área de Ciência da Informação, no intuito de favorecer uma melhor compreensão de tais tecnologias, seus reflexos e aplicações na área de Ciência da Informação. Conclusão: Constatou-se que atualmente a linguagem XML apresenta um maior número de aplicações no cotidiano dos profissionais da informação, impulsionando grandes avanços para a área, contudo, RDF ainda é pouco explorada, evidenciando a necessidade de um melhor entendimento da evolução das linguagens de marcação e dos conceitos subjacentes, de modo que tais avanços tecnológicos não sejam desenvolvidos a partir de um 'vazio conceitual', com o único

\footnotetext{
${ }^{1}$ Doutor em Ciência da Informação pela Faculdade de Filosofia e Ciências da Universidade Estadual Paulista (UNESP), campus de Marília. Docente do Programa de Pós-Graduação em Ciência da Informação da Universidade Federal de São Carlos (UFSCar). E-mail: ramalho@ufscar.br

${ }^{2}$ Mestrando do Programa de Pós-Graduação em Ciência da Informação da Universidade Federal de São Carlos (UFSCar). E-mail: pgeorgemm22@gmail.com

${ }^{3}$ Mestrando do Programa de Pós-Graduação em Ciência da Informação da Universidade Federal de São Carlos (UFSCar). Bolsista FAPESP. E-mail: janailtonlopes20@gmail.com
} 
objetivo de atender demandas de mercado, e possam ser devidamente sedimentados no campo teórico.

Descritores: Tecnologias da Informação. Linguagens de Marcação. Modelo de Dados. Representação da Informação. Web Semântica.

\section{INTRODUÇÃO}

As denominadas Linguagens de Marcação sempre tiveram relação direta com os instrumentos e práticas profissionais identificados no campo da Ciência da informação, a partir do desenvolvimento do ambiente Web tais tecnologias ganharam maior destaque, impulsionado a criação de uma grande variedade de aplicações com diferentes características e propósitos.

No âmbito nacional pesquisadores da Escola de Ciência da Informação da Universidade Federal de Minas Gerais (UFMG) foram pioneiros em relação às publicações direcionadas ao estudo das Linguagens de Marcação na área de Ciência da Informação, destacando as potencialidades desta temática, conforme descreve BAX (2001, p. 37)

[...] a emergência de estruturas de dados mais ricamente anotadas (ou marcadas) pode ser o catalisador que falta para a concepção de novas aplicações que promoverão 0 armazenamento, compartilhamento e processamento de conhecimento.

Almeida (2002, p. 5) ao se referir ao estudo das Linguagens de Marcação também apresenta como "[...] um campo fértil para discussões, proposições e estudo por profissionais da Ciência da Informação."

Após quase duas décadas das referidas publicações muitos avanços podem ser identificados nesta temática e seu crescente impacto na área de Ciência da Informação. Assim, o objetivo deste trabalho é apresentar um compêndio da evolução das linguagens de marcação, analisando tais tecnologias sob o enfoque da área de Ciência da Informação e descrever os conceitos e características das principais linguagens utilizadas na área.

Trata-se de uma pesquisa de cunho teórico, realizada a partir de uma revisão de literatura sobre a evolução das linguagens de marcação sob o 
enfoque da Ciência da informação e abordagem qualitativa, pois busca apresentar características voltadas à descrição, compreensão e explicação de relações de um determinado fenômeno de modo a considerar o caráter interativo entre os objetivos definidos e as orientações teóricas da pesquisa. Caracteriza-se, também, como pesquisa de natureza aplicada, uma vez que "[...] objetiva gerar conhecimentos para aplicação prática, dirigidos à solução de problemas específicos." (SILVEIRA; CÓRDOVA, 2009, p. 35).

\section{ORIGEM DAS LINGUAGENS DE MARCAÇÃO}

O conceito de Linguagem é utilizado tanto para se referir a capacidade humana de aquisição e utilização de sistemas complexos de comunicação, como também para qualquer meio sistemático de comunicação que se utiliza de signos para troca de mensagens.

Com o desenvolvimento das tecnologias surgiu o conceito de Linguagem Computacional que pode ser definida sucintamente como um conjunto de símbolos e regras formais utilizadas para a definição de sentenças que representam e processam instruções para computadores. Entre os diversos tipos de linguagens computacionais existentes este artigo tem como foco as denominadas Linguagens de Marcação, um tipo específico de linguagem computacional que utiliza marcações <tags> para descrever e estruturar recursos informacionais.

A utilização de marcações na elaboração de materiais tipográficos antecede o surgimento dos computadores digitais, antes da era das publicações digitais as anotações, estilos e estruturas das publicações eram inseridas manualmente sobre a margem ou juntamente com o texto. Contudo, iremos aqui nos focar na evolução das denominadas Linguagens de Marcação utilizadas computacionalmente.

Historicamente, os primeiros documentos eletrônicos continham códigos de controle ou macros específicos, o que exigia que cada arquivo fosse interpretado e formatado de maneira independente. Apenas no final da década de 1960 que o conceito de 'codificação genérica' começou a ser difundido, tendo 
como base a definição de marcas de texto, onde cada código é associado a um conjunto de instruções de formatação, possibilitando a identificação dos elementos de um documento, como cabeçalhos, parágrafos, legendas e ilustrações.

O início do movimento de 'codificação genérica' é creditado a William Tunnicliffe que em 1967, durante uma reunião no escritório de imprensa do governo do Canadá, fez uma apresentação abordando a separação do conteúdo informacional do formato dos documentos. Em 1969 um designer de livros chamado Stanley Rice propôs a ideia de um catálogo universal de tags de 'estrutura editorial' parametrizadas. Assim, Norman Scharpf, então diretor da associação norte-americana Graphic Communications Association (GCA) reconheceu a importância dessas tendências e estabeleceu um projeto genérico de codificação para a indústria editorial, que culminou no desenvolvimento de um padrão denominado como GenCode.

Em paralelo às pesquisas desenvolvidas pela GCA, na tentativa de resolver os problemas internos de publicação eletrônica, a empresa International Business Machines (IBM) também desenvolvia pesquisas relacionadas a padronização de marcações que pudessem favorecer um melhor processamento automatizado dos documentos eletrônicos. Assim, em 1969 os pesquisadores Charles Goldfarb, Edward Mosher e Raymond Lorie, publicaram a primeira versão da linguagem Generalized Markup Language (GML), considerada como a primeira Linguagem de Marcação amplamente reconhecida.

A partir da disseminação do uso dos computadores proliferam as áreas de utilização e consequentemente os tipos de documentos, de modo que cada documento possuía requisitos diferentes, exigindo conjuntos específicos de anotações. Tal cenário evidenciou a necessidade de padronizar a forma de inserir, processar e manipular as anotações dos documentos.

Nesta perspectiva, em 1978 representantes responsáveis pela definição do padrão GenCode e da linguagem GML se uniram formando um comitê da American National Standards Institute (ANSI), com o objetivo de desenvolver uma Linguagem de marcação padrão para a especificação de anotações em documentos digitais, a qual foi denominada como Standard Generalized Markup 
Language (SGML), dando origem em 1986 ao padrão ISO 8879/86 (EDWARDS, 1997; SENGUPTA; DILLON, 1997; MILLER JUNIOR, 2003).

SGML foi concebida como uma metalinguagem, linguagem que possibilita a definição de outras linguagens de marcação a partir da descrição formal de regras sintáticas para a definição de sistemas de marcação textual, onde cada sistema de marcação é especificado, essencialmente, por uma gramática livre de contexto.

Amplamente difundida, a linguagem SGML logo passou a ser utilizado em grande escala em projetos governamentais e comerciais para 0 compartilhamento de documentos que pudessem ser legíveis por máquinas. Com tais características, uma das grandes vantagens da criação da SGML, à época, era a possibilidade de descrever documentos a partir de sua própria gramática (TEIXEIRA, 2002; MORENO; BRASCHER, 2007).

Almeida (2002, p. 6) destaca que um dos principais objetivos com a criação do SGML "[...] é garantir que documentos codificados de acordo com suas regras possam ser transportados de um ambiente de hardware e software para outro, sem perda de informação."

Cada documento de SGML carrega consigo a especificação de sua própria gramática formal, possibilitando que por meio de um Document Type Definition (DTD) os sistemas computacionais interpretem as marcações existentes.

No campo da Ciência da Informação a linguagem SGML possibilitou a representação padronizada dos documentos de maneira estruturada, a partir da utilização de marcações passíveis de serem processadas por sistemas computacionais, independente do sistema operacional ou software utilizado, favorecendo uma maior abrangência do campo de atuação dos profissionais da informação. Contudo, a complexidade da SGML dificultava sua popularização, influenciando a criação de nichos profissionais especializados em aplicações específicas deste padrão em detrimento a utilizações gerais.

Entre as aplicações mais populares da SGML podemos destacar a HyperText Markup Language (HTML), criada no final da década de 1980 pelo pesquisador Berners-Lee tornou-se um dos principais pilares para a concepção 
da Word Wide Web, dando origem a uma nova forma de visualização da Internet, incorporando o uso de links em documentos hipertextuais.

\subsection{Hypertext Markup Language (HTML)}

A HyperText Markup Language (HTML) é uma linguagem baseada na SGML e foi a primeira linguagem de marcação que se tornou popular, devido principalmente a facilidade de sua utilização. HTML utiliza um conjunto limitado de marcações pré-definidas em sua DTD, possibilitando apresentar de maneira simples os diferentes tipos de recursos informacionais em uma página Web.

Marcondes (2007) destaca que uma das principais características da linguagem HTML é sua estrutura flexível e de fácil manuseio, possibilitando descrever os elementos que compõem o documento e a forma de sua apresentação, sendo considerada a linguagem de marcação mais simples e fácil de ser utilizada.

Cada uma das versões de HTML se fundamenta em um novo DTD, limitando-se às marcações previamente definidas. Almeida (2002) destaca que, o uso da HTML para a representação de páginas Web possibilitou que os dados fossem apresentados em uma estrutura simples e de fácil leitura. Contudo, sua concepção baseada em marcações fixas trouxe limitações para a representação de informações uma vez que, sendo uma linguagem de marcação voltada, principalmente, para a apresentação dos documentos em ambientes digitais, HTML apenas define a forma como a informação é exibida não se preocupando com o significado da palavra.

Conforme destacam Alesso e Smith (2005) a linguagem HTML tem como foco principal a apresentação dos documentos, e em suas primeiras versões não fornecia nenhum tipo de suporte para a representação semântica dos conteúdos. Apenas em outubro de 2014 o W3C disponibilizou a especificação HTML5, incorporando novas funcionalidades a Linguagem HTML, e apresenta a totalidade da tecnologia aplicada na linguagem HTML (HICKSON et al., 2014). 
No âmbito da área da Ciência da Informação a utilização da linguagem HTML influenciou o desenvolvimento de novos meios de representação e disseminação de informações em ambientes digitais, contribuindo fortemente para a popularização dos catálogos on-line, denominados como Online Public Access Catalog (OPAC), pois a facilidade de publicar conteúdos em HTML favoreceu a disseminação dos acervos na Web.

\section{2 eXtensible Markup Language (XML)}

Criada pelo engenheiro Jon Bosak, em 1996, a eXtensible Markup Language ( $\mathrm{XML}$ ) foi desenvolvida com base na linguagem SGML e teve sua recomendação divulgada em 1998. Diferente da linguagem HTML, que se caracteriza como um esquema de aplicação SGML a linguagem XML é um subconjunto simplificado da SGML.

Deste modo, a linguagem XML também é considerada como uma metalinguagem de marcação, pois permite a criação de novas linguagens a partir da definição vocabulários personalizados, favorecendo o uso de marcações personalizadas que podem ser definidas pelo próprio desenvolvedor (BAX, 2001; HEITLINGER, 2001; RAMALHO, 2006).

Conforme destaca Almeida (2002, p. 7), "[...] a especificação do XML define um dialeto simples do SGML, permitindo o processamento dos documentos na Internet e utilizando-se de recursos inexistentes no HTML." Segundo Furgeri (2006), uma das funções básicas de XML é se ocupar com a representação de informações de forma estruturada, pois diferente de HTML onde as marcações definem a forma de apresentação, em XML as marcações são utilizadas para descrever a estrutura dos conteúdos.

$\mathrm{Na}$ época de sua criação um dos fatores que dificultaram a aceitação da linguagem XML foi uma concepção equivocada de que a mesma fosse uma linguagem que viria a substituir HTML. Contudo, tais linguagens possuem propósitos distintos, enquanto HTML tem como foco a representação dos documentos como páginas Web, XML favorece a representação formal dos 
elementos que compõem os documentos, de modo que tal estrutura possa ser processada computacionalmente (RAMALHO, 2006).

Pode-se afirmar que XML desempenha um papel semelhante ao de uma 'linguagem universal' podendo ser utilizada para o desenvolvimento de diferentes linguagens de uso específico, favorecendo a interoperabilidade entre sistemas, por meio da simplificação das comunicações entre plataformas não heterogêneas (DAUM; MERTEN, 2002; OPARA; SRIVASTAVA, 2003).

A linguagem $X M L$ por se tratar de um subconjunto simplificado da SGML permite a formalização de vocabulários para especificar os tipos de dados de um documento, comumente denominados como esquemas. Tradicionalmente existia apenas o padrão denominado Document Type Definition (DTD) para a definição de esquemas XML, apenas em 2001 foi publicado um novo padrão denominado XML Schema Definition (XSD), ou simplesmente XML Schema, desenvolvido com o objetivo de superar as limitações existentes no DTD e facilitar a codificação de esquemas utilizando a mesma sintaxe da linguagem XML.

Os esquemas são constituídos por um conjunto de elementos, que podem ser do tipo simples ou complexo, e consistem na representação de vocabulários específicos, por meio da descrição formal dos elementos, estrutura e tipos de dados esperados. Atualmente o padrão XSD é o mais utilizado, contudo muitas implementações tradicionais foram elaboradas com base no padrão DTD e até hoje são utilizadas.

$\mathrm{Na}$ área de Ciência da Informação a linguagem XML passou a ser utilizada para representação formal de padrões de metadados, os padrões MARCXML e Dublin Core são dois exemplos de padrões codificados em XML amplamente conhecidos. Computacionalmente podemos afirmar que tanto 0 MARCXML como o Dublin Core consistem em esquemas XML que definem formalmente quais são os elementos, atributos e formatos de dados esperados em cada um dos campos dos metadados, favorecendo uma maior interoperabilidade.

Apesar de XML ter representando um grande avanço em relação a forma como representamos conteúdos informacionais no ambiente Web, ao 
longo dos últimos anos, verificou-se uma crescente demanda na descrição formal das relações existentes entre os recursos heterogêneos, dando origem ao Resource Description Framework.

\subsection{Resource Description Framework (RDF)}

Resource Description Framework (RDF) é uma linguagem declarativa, que se utiliza de marcações definidas em XML para representar sentenças sobre propriedades e relacionamentos entre recursos na Web. Conforme destacam Lassila e Swick (1999, não paginado, tradução nossa).

RDF é uma aplicação da linguagem XML que se propõe ser uma base para o processamento de metadados na Web. Sua padronização estabelece um modelo de dados e sintaxe para codificar, representar e transmitir metadados, com o objetivo de torná-los processáveis por máquina, promovendo a integração dos sistemas de informação disponíveis na Web.

Desenvolvida em 1997 pelos editores Ora Lassila e Ralph Swick o RDF foi projetado para trabalhar com metadados que promovem o intercâmbio de informações entre processos automatizados sem perda de significado (ALESSO; SMITH, 2005; FURGERI, 2006).

Diferente da linguagem $\mathrm{XML}$ que visa descrever formalmente os elementos e a estrutura de um recurso informacional, RDF se propõe a interligar diferentes recursos a partir de declarações que possibilitem formalizar os relacionamentos existentes.

RDF permite descrever declarações a respeito de recursos, não exigindo modificações nos mesmos, de modo que uma declaração RDF é uma entidade separada do recurso ao qual ela se refere, podendo inclusive constituir outro recurso. Nesta perspectiva, pode haver muitas declarações RDF distribuídas pela Web referindo-se ao mesmo recurso, descrevendo diferentes propriedades, ou mesmo contextualizando-o a partir de domínios diferentes.

Segundo a recomendação do W3C, publicada por Klyne et al.(2004), os princípios fundamentais do padrão RDF baseiam-se na tripla 'Subject - Predicate - Object'. De modo que 'Subject' é o recurso ao qual uma sentença está se 
referindo, 'Predicate' descreve uma característica, propriedade, ou relacionamento utilizado para descrever algo sobre este recurso e 'Object' é o valor de uma determinada característica do recurso referenciado, podendo inclusive ser outro recurso.

Por se caracterizar como um modelo de dados abstrato as declarações RDF podem ser representadas de diversas formas, denominadas como tipos de serialização, desde que a representação permaneça fiel às suas propriedades abstratas. Assim, é possível representar graficamente uma declaração RDF a partir de um grafo rotulado direcionado, também denominado como 'diagramas de nós e arcos', por convenção os recursos são representados como elipses, os valores literais como retângulos e os predicados utilizando arcos direcionados do recurso (sujeito) para o valor (objeto), conforme apresentado na figura 1.

Figura 1: Grafo de uma declaração RDF

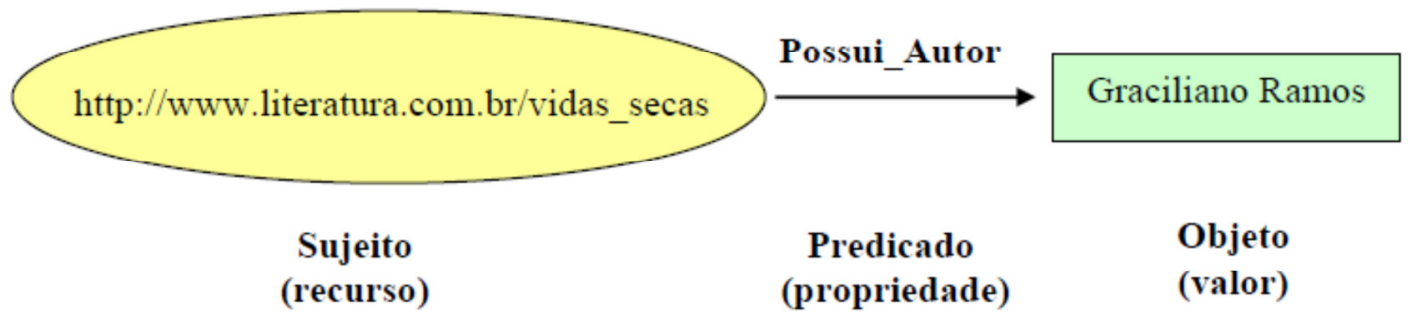

Fonte: Elaborado pelos autores

As triplas RDF possibilitam descrever formalmente classes, propriedades e relacionamentos, expressar a semântica básica de uma declaração a partir de um vocabulário RDF. Assim, RDF é considerado com a tecnologia base para a criação de ontologias, embora possua limitações no suporte à realização de inferências.

Declarações em RDF possibilitam a automação de inferências simples, contribuindo para maior interoperabilidade, uma vez que permitem estabelecer relações formais entre informações disponíveis em diferentes domínios (LASSILA; SWICK, 1999; KONTOPOULOS; KRAVARI; BASSILIADES, 2007; BISCHOF et al., 2011). 
$\mathrm{Na}$ área de Ciência da Informação RDF tem sido utilizado em aplicações relacionadas à representação de conteúdos informacionais em ambientes digitais, contribuindo para uma disseminação informacional mais eficiente e eficaz (ALEIXO, SANT'ANA; RAMALHO, 2015).

Ao longo da última década novos instrumentos de modelagem, representação e organização de recursos informacionais têm sido propostos baseados em RDF, entre os quais pode-se destacar o Bibliographic Framework (BIBFRAME) um modelo de dados lançado oficialmente em maio de 2011 a partir de uma iniciativa da Library of Congress (EUA) com o objetivo de revistar e, a longo prazo, implementar um novo padrão para a descrição de recursos bibliográficos (RAMALHO, 2015).

Apesar do estado ainda incipiente do BIBIFRAME e do número ainda limitado de aplicações baseadas em RDF no ambiento da área de Ciência da Informação verifica-se que tal tecnologia vai ao encontro das novas tendências de pesquisas como: Linked Data; Data Science; Publicação Ampliada e Web Semântica.

\section{CONSIDERAÇÕES FINAIS}

O presente artigo apresenta um breve compêndio da evolução das linguagens de marcação, contribuindo para uma maior discussão desta temática na área de Ciência da Informação, no intuito de favorecer uma melhor compreensão de tais tecnologias, seus reflexos e aplicações na área de Ciência da Informação.

Observa-se que as Linguagens de Marcação sempre tiveram relação direta com os instrumentos e práticas profissionais identificados no campo da Ciência da informação, favorecendo melhorias nos processos de representação e estruturação de recursos informacionais.

Ao longo da última década a Linguagem XML é a que apresenta um maior número de aplicações no cotidiano dos profissionais da informação, contudo ainda se observa que muitos profissionais da área possuem pouca familiaridade 
com esta tecnologia, dificultando o correto entendimento, uso e desenvolvimento de novos instrumentos de representação.

Em uma sociedade cada vez mais baseada em interações virtuais tornase imperativo a necessidade de tecnologias como RDF que possibilitem descrever formalmente os relacionamentos existentes entre recursos informacionais, contudo tal linguagem ainda é pouco explorada na área de Ciência da informação, evidenciando a necessidade de um melhor entendimento da evolução das linguagens de marcação e dos conceitos que fundamentam tais tecnologias, para que pesquisas que abordem temas como Linked Data; Data Science; Publicação Ampliada e Web Semântica possam realmente ser fundamentadas nos princípios da área de Ciência da Informação, fornecendo subsídios teóricos para que tais tecnologias não sejam desenvolvidas a partir de um 'vazio conceitual', com o único objetivo de atender demandas de mercado, de modo que os avanços tecnológicos possam ser devidamente sedimentados no campo teórico.

\section{REFERÊNCIAS}

ALEIXO, D. V. B.S.; SANT'ANA, R. C. G.; RAMALHO, R. A. S. Resource description framework (RDF): o impacto nas publicações na Ciência da Informação. 2017. In: SEMINÁRIO EM CIÊNCIA DA INFORMAÇÃO, 7., 2017. Londrina. Anais eletrônicos...Londrina: UEL, 2017. Disponível em: <http://www.uel.br/eventos/cinf/index.php/secin2017/secin2107/paper/viewFile/ 472/32>. Acesso em: 08 nov. 2017.

\section{ALESSO, H. P.; SMITH, C. F. Developing Semantic Web services.} Massachusetts: A K Peters, Ltd, 2005.

ALMEIDA, M. B. Uma introdução ao XML, sua utilização na internet e alguns conceitos complementares. Ciência da Informação, Brasília, v. 31, n. 2, p. 513, maio/ago. 2002.

BAX, M. P. Introdução às linguagens de marcas. Ciência da Informação, v. 30, n. 1, p. 32-38, 2001. Disponível em: <http://www.brapci.ufpr.br/brapci/v/a/650>. Acesso em: 11 set. 2016.

BISCHOF, S. et al. Mapping between RDF and XML with XSPARQL. 2011. Disponível em: <http://link.springer.com/article/10.1007/s13740-012-0008-7>. Acesso em: 11 fev. 2017. 
DAUM, B.; MERTEN, U. Arquitetura de sistemas com XML: conteúdo, processo e apresentação. Rio de Janeiro: Campus, 2002, p. 43-48.

EDWARDS, M. XML: data the way you want it. [S. I.]: Microsoft, 1997.

FURGERI, S. O papel das linguagens de marcação para a Ciência da Informação. TransInformação, Campinas, v. 18, n. 3, p. 225-239, set./dez. 2006. Disponível em: <http://periodicos.puccampinas.edu.br/seer/index.php/transinfo/article/view/670>. Acesso em: 18 mar. 2017.

HEITLINGER, P. O guia prático da XML: conceitos, exemplos, prática e aplicações da linguagem universal. 2001. Disponível em:

<http://www.centroatl.pt/titulos/tecnologias/imagens/oguiapraticoda-xmlexcerto.pdf>. Acesso em: 25 ago. 2016.

HICKSON, I. et al. HTML5: a vocabulary and associated APIs for HTML and XHTML.W3C Recommendation. 2014. Disponível em: $<$ https://www.w3.org/TR/html5/single-page.html>. Acesso em: 21 jan. 2016.

KLYNE, G. et al. Resource Description Framework: concepts and abstract syntax. W3C recommendation. 2004. Disponível em: <http://www.w3.org/TR/2004/REC-rdf-concepts-20040210 >. Acesso em: 15 set. 2015.

KONTOPOULOS, E.; KRAVARI, K.; BASSILIADES, N. Object-Oriented modeling of RDF schema ontologies. 2007. Disponível em:

$<$ http://pis.csd.auth.gr/publications/pci2007-kontopo_et_al.pdf>. Acesso em: 24 jan. 2017.

LASSILA, O; SWICK, R. Resource Description Framework (RDF): model and syntax specification. W3C Recommendation. [S.I. : s.n.],1999. Disponível em: <https://www.w3.org/TR/1999/REC-rdf-syntax-19990222/>. Acesso em: 09 mar. 2017.

MARCONDES, C. A. HTML 4.0 fundamental: a base da programação para a web. 2. ed. São Paulo: Érica, 2007.

MILLER JUNIOR, R. Teoria e problemas de XML. Porto Alegre: Bookman, 2003.

MORENO, F. P.; BRASCHER, M. MARC, MARCXML e FRBR: relações encontradas na literatura. Inf. \& Soc.: Est., João Pessoa, v.17, n.3, p. 14-16, 2007. Disponível em:

<http://www.ies.ufpb.br/ojs2/index.php/ies/article/view/834/1582> Acesso em: 07 abr. 2015. 
OPARA, E.; SRIVASTAVA, M. Extensible Markup Language: an enterprise integration web delivery system. Communications of the IIMA, v. 3: n. 2, p. 5, 2003. Disponível em: <http://scholarworks.lib.csusb.edu/ciima/vol3/iss2/5>. Acesso em: 12 fev. 2017.

RAMALHO, R. A. S. Web Semântica: aspectos interdisciplinares da gestão de recursos informacionais no âmbito da Ciência da Informação. 2006, 120 f. Dissertação (Mestrado em Ciência da Informação) - Faculdade de Filosofia e Ciências, Universidade Estadual Paulista, Marília, 2006.

RAMALHO, R.A.S. Análise do Modelo de Dados SKOS: Sistema de Organização do Conhecimento Simples para a Web. Informação \& Tecnologia (Itec), v. 2, p. 66-79, 2015.

SILVEIRA, D. T.; CÓRDOVA, F. P. A pesquisa científica. In: GERHARDT, T. E.; SILVEIRA, D. T. (Orgs.). Métodos de pesquisa. Porto Alegre: Editora da UFRGS, 2009.

SENGUPTA, A.; DILLON, A. Extending SGML to accommodate database functions: a methodological overview. Journal of the American Society for Information Science, v. 48, n. 7, p. 629-637, 1997. Disponível em: <http://hdl.handle.net/10150/105183>. Acesso em: 17 fev. 2017.

TEIXEIRA, E. C. Utilizando XML para publicação de dados multimídia na Web. 2002. 77 f. Dissertação (Mestrado em Ciência da Computação) Universidade Federal de São Carlos, São Carlos, 2002. Disponível em: <https://repositorio.ufscar.br/handle/ufscar/527>. Acesso em: 15 jan. 2017.

\title{
EVOLUTION OF MARKUP LANGUAGES: A BRIEF HISTORY IN LIGHT OF INFORMATION SCIENCE
}

\begin{abstract}
Introduction: The so-called Markup Languages have always been directly related to the professional practices identified in the field of Information Science, from the development of the Web environment, such technologies have gained greater prominence, boosting the creation of a wide variety of applications with different characteristics and purposes.

Objective: To present a compendium of the evolution of markup languages, analyzing such technologies under the focus of Information Science and describing the concepts and characteristics of the main languages used in the area. Methodology: Review of literature on the evolution of markup languages from the perspective of information science, qualitative approach of applied nature. Results: The article presents a brief review on the evolution of markup languages, contributing to a greater discussion of this topic in the area of Information Science, in order to favor a better understanding of these technologies, their reflexes and applications in the area of Science of
\end{abstract}


Information. Conclusion: It was verified that currently $\mathrm{XML}$ is tha language with presents a greater number of applications in the daily life of information professionals, impelling great advances in the area, however, RDF is still little explored, evidencing the need for a better understanding of the evolution of the languages of marking and underlying concepts, so that such technologies are not developed from a 'conceptual vacuum', with the sole objective of meeting market demands, for such technologies to be properly consolidated in the theoretical field.

Descriptors: Information Technology. Markup Languages. Data Modeling. Information Representation. Semantic Web.

\title{
EVOLUCIÓN DE LOS LENGUAJES DE MARCADO: UN BREVE HISTÓRICO A LA LUZ DE LA CIENCIA DE LA INFORMACIÓN
}

\begin{abstract}
RESUMEN
Introducción: Las denominadas Lenguajes de Marcación siempre tuvieron relación directa con las prácticas profesionales identificadas en el campo de la Ciencia de la información, a partir del desarrollo del ambiente Web tales tecnologías ganaron mayor destaque, impulsando la creación de una gran variedad de aplicaciones con diferentes características y propósitos. Objetivo: Presentar un compendio de la evolución de los lenguajes de marcado, analizando tales tecnologías bajo el enfoque del área de Ciencia de la Información y describir los conceptos y características de los principales lenguajes utilizados en el área. Metodología: Revisión de literatura sobre evolución de los lenguajes de marcado, basado en el enfoque de la Ciencia de la información, abordaje cualitativo de naturaleza aplicada. Resultados: El artículo presenta una breve revisión sobre la evolución de los lenguajes de marcado, contribuyendo a una mayor discusión de esta temática en el área de Ciencia de la Información, con el fin de favorecer una mejor comprensión de tales tecnologías, sus reflejos y aplicaciones en el área de Ciencia de la Ciencia información. Conclusión: Se constató que actualmente el lenguaje XML presenta un mayor número de aplicaciones en el cotidiano de los profesionales de la información, impulsando grandes avances hacia el área, sin embargo, RDF todavía es poco explorada, evidenciando la necesidad de un mejor entendimiento de la evolución de los lenguajes de los lenguajes la marca y los conceptos subyacentes, de modo que tales tecnologías no se desarrollen a partir de un 'vacío conceptual', con el único objetivo de atender demandas de mercado, y puedan ser debidamente sedimentados en el campo teórico.
\end{abstract}

Descriptores: Tecnologías de Información. Lenguajes de Marcado. Modelado de datos. Representación de la información. Web Semántica.

Recebido: 20.10 .2017

Aceito: 28.12.2017

Inf. Prof., Londrina, v. 6, n. 2, p. 20 - 34, jul./dez. 2017

http://www.uel.br/revistas/infoprof/ 